\title{
Prevalence of anti-neutrophil antibody in primary sclerosing cholangitis and ulcerative colitis using an alkaline phosphatase technique
}

\author{
S K Lo, K A Fleming, R W Chapman
}

\begin{abstract}
The detection of a nuclear anti-neutrophil antibody in patients with primary sclerosing cholangitis (PSC), using an immunoperoxidase technique, was recently reported by us. Subsequently, detection of a cytoplasmic antineutrophil antibody was reported by others, using a two stage procedure of enzyme linked immunosorbent assay followed by an immunofluorescent method. Detection of cytoplasmic anti-neutrophil antibody in PSC, which, in contrast to that two stage procedure, uses a simple one step immuno-alkaline phosphatase method is now reported. Normal human neutrophils were cytocentrifuged, ethanol fixed, and then incubated with coded patients' sera. Rabbit anti-human immunoglobulin conjugated with alkaline phosphatase was used to detect the bound antibody. Fast red was used to visualise the reaction. Twenty three of $\mathbf{3 0}$ (77\%) PSC patients showed positive granular cytoplasmic staining (with some perinuclear accentuation) with a network of cytoplasmic filaments. Fifteen of $45(33 \%)$ ulcerative colitis patients and 1 of 3 chronic active hepatitis patients showed similar staining. Thirty five of 152 patients with ulcerative colitis, chronic active hepatitis, and a variety of other liver diseases showed a different pattern of cytoplasmic labelling, with no surrounding filaments. Seventy nine patients, including seven PSC patients and 33 normal subjects, were negative. In comparison, $86 \%$ of PSC patients, $\mathbf{5 7 \%}$ of patients with primary biliary cirrhosis, $50 \%$ of normal subjects, and well over $60 \%$ of patients with ulcerative colitis, Crohn's disease, alcoholic liver disease, and chronic active hepatitis were positive using the one step immunofluorescent method. This method is more specific for PSC than those described in recent reports and may be of diagnostic importance.

(Gut 1992; 33: 1370-1375)
\end{abstract}

Department of

Gastroenterology, John

Radcliffe Hospital,

Oxford OX3 9DU

S K Lo

R W Chapman

Nuffield Department of Pathology and

Bacteriology, University of Oxford, John Radcliffe Hospital, Oxford K A Fleming

Correspondence to: Dr R W Chapman

Accepted for publication 6 January 1992
Primary sclerosing cholangitis (PSC) is a chronic cholestatic liver disease that is characterised by inflammation and fibrosis of both intra- and extrahepatic bile ducts. This can progress to bile duct destruction, biliary cirrhosis, and sometimes cholangiocarcinoma. Although the aetiology of PSC is unknown, recent evidence suggests that immune mechanisms may play an important role in its pathogenesis. This evidence includes an increased prevalence of HLA B8 DR3 haplotype.' an aberrant expression of HLA increased $\mathrm{CD} 4 / \mathrm{CD} 8$ ratio of circulating $\mathrm{T}$ cells DR antigen in intrahepatic bile ducts,' and an and increased portal tract $\mathrm{T}$ lymphocytes. Moreover, we have previously reported detection of a nuclear anti-neutrophil antibody in $80 \%$ of PSC patients, ${ }^{+}$although it was also present in some other liver diseases. A recent study which used the western blotting technique also showed an antibody against granulocytes in similar patients. ${ }^{5}$ Furthermore, a cytoplasmic antineutrophil antibody has also been detected in PSC $^{67}$ and ulcerative colitis ${ }^{8-12}$ using combined indirect immunofluorescent staining with or without ELISA. However, the specificity of this antibody for PSC, as opposed to ulcerative colitis, is unclear and a recent study has shown that the immunofluorescent technique is less specific and less sensitive than previously demonstrated by Duerr et al. ${ }^{13}$ Using a technique different to that used in our original report, we have now detected an antibody against the cytoplasm of neutrophils. This antibody seems more specific for PSC than the other autoantibodies previously reported.

\section{Methods}

PATIENTS

PSC

Serum samples from 30 PSC patients were studied. The diagnosis of PSC was established by clinical, histological, and cholangiographic criteria. ${ }^{1+}$ The mean age of the group was $53 \cdot 4$ years with a range of 24-82 years. Twenty patients were male. Thirteen patients were graded as I and II on Ludwig's criteria, ${ }^{15}$ three patients were cirrhotic and four were normal. Twenty five of the PSC patients had coexistent ulcerative colitis while three had Crohn's disease. The mean duration of the disease was $6 \cdot 1$ years (range 8 months- 14 years). Fourteen of the 30 PSC patients had the HLA B8 DR3 haplotype on serotyping (NIH lymphocytotoxicity assay). The clinical details are shown in Table I.

\section{Inflammatory bowel disease}

Seventy seven patients with inflammatory bowel disease were also included in the study. Of these, 45 patients had ulcerative colitis (age range: 18-74 with a mean age of $48 \cdot 7$ ). There were 22 men. All but two patients were on some form of maintenance therapy (sulphasalazine/ mesalazine). Nineteen were also on steroids with or without azathioprine. Twenty two had active colitis at the time serum was obtained. Thirty 
TABLE I Details of primary sclerosing cholangitis patients $(n=30)$

\begin{tabular}{lc}
\hline Male/female & $20 / 10$ \\
Age (yr): mean (range) & $53 \cdot 4(24-82)$ \\
Duration of disease: mean (range) & $6 \cdot 1$ years (8 months-14 years) \\
IBD & 25 \\
$\quad$ Ulcerative colitis & 3 \\
Crohn's disease & 14 \\
HLA B8 DR3 & \\
Liver histology: & 4 \\
Normal & 6 \\
Stage 1 & 7 \\
Stage 2 & 6 \\
Stage 3 & 3 \\
Stage 4 & 4 \\
Unknown & \\
\hline
\end{tabular}

$\mathrm{IBD}=$ inflammatory bowel disease

two patients had Crohn's disease (16 had colitis). Their mean age was 46.6 years with a range of 21-82 years, and 10 were men. All had normal liver function tests.

\section{Other diseases}

Serum samples were also obtained from other groups of patients; 18 with alcoholic liver disease, 14 with primary biliary cirrhosis, six with large duct obstructive jaundice, three with autoimmune chronic active hepatitis, two with haemochromatosis, and two with coeliac disease. All the above diagnoses were based on standard criteria. Thirty five healthy subjects with different blood groups were also included in the study. A further eight patients with Wegener's granulomatosis who were known to have circulating anti-neutrophil cytoplasmic antibody (ANCA) as determined by the standard immunofluorescent method ${ }^{16}$ were also tested.

\section{METHODS}

Indirect immunohistological staining using alkaline phosphatase

Normal human neutrophils were harvested from peripheral blood using Mono-Poly Resolving Medium (Flow Laboratories). ${ }^{17}$ The neutrophils were washed with TBS (50 mM Tris, $145 \mathrm{mM}$

Figure 1: Type 1 pattern of staining. Note the granular cytoplasm with perinuclear accentuation (big arrow) and the filaments around the cells (small arrows).
Culture Services, UK). They were then washed by centrifugation ( $300 \mathrm{~g}$ for 10 minutes) and resuspended in the same buffer and fetal calf serum. Aliquots of suspended cells were cytocentrifuged (Shandon Southern Cytocentrifuge, Cheshire, England) at $500 \mathrm{rpm}$ for 5 minutes onto slides. Cells were air dried and then fixed with absolute alcohol for 5 minutes. These cells were then incubated with coded patient's serum, diluted 1:10 in TBS for 30 minutes. After washing with TBS for 1 minute, bound antibody was detected using indirect immunohistochemistry. Rabbit antibody to human immunoglobulin heavy chains (IgA/G/M) (DAKO, High Wycombe, UK) conjugated with alkaline phosphatase was used as a secondary antibody diluted 1:30 in TBS. Fast red TR salt (Sigma) was used as a substrate for alkaline phosphatase. All procedures were carried out at room temperature.

\section{Indirect immunohistological staining using} peroxidase

Similar procedures were carried out as described above except that the secondary antibody was rabbit antibody to human immunoglobulin conjugated with peroxidase (DAKO, High Wycombe, UK) instead of alkaline phosphatase. The substrate was 3,3'-diaminobenzidine hydrochloride (Sigma) $(6 \mathrm{mg}$ in $10 \mathrm{ml} \mathrm{0.05} \mathrm{M}$ Tris buffer, $\mathrm{pH} 7 \cdot 6$ ) and hydrogen peroxide (3\%). This test was performed on sera from patients with PSC, ulcerative colitis, and Crohn's disease.

\section{Indirect immunohistological staining using}

fluorescein conjugated antibody

Exactly the same procedure was carried out as described above. The secondary antibody used was rabbit antibody to human immunoglobulin heavy chains conjugated with fluorescein isothiocynate isomer 1 (FITC) (DAKO, High Wycombe, UK). Slides were examined by fluorescence microscope.

Sodium-dodecyl-sulfate (SDS) polyacrylamide gel electrophoresis

Neutrophils were harvested as described earlier. After washing with TBS, the cells were resuspended in $1.5 \mathrm{ml}$ of $0.34 \mathrm{M}$ sucrose containing $1 \mathrm{mM}$ iodoacetamide, $0 \cdot 1 \mathrm{M}$ phenylmethylsulphonylfluoride, $1 \mu \mathrm{M}$ leupeptin, and Pepstatin A (Sigma). The suspension was sonicated for 1 minute at $0^{\circ} \mathrm{C}$.

SDS electrophoresis was carried out with a $5.5 \%$ stacking and a $10 \%$ running gel according to Laemmli's method ${ }^{18}$ with some modification.

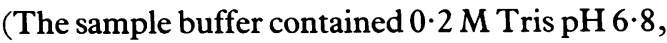
2\% SDS, $8 \mathrm{M}$ urea and dithiothreitol (Sigma) $(6 \mathrm{mg} / \mathrm{ml}$ of sample buffer.))

\section{Western blot}

Proteins were transferred electrophoretically by western blotting ${ }^{19}$ on to Immobilon sheets (Millipore) using semi-dry apparatus. After blocking free protein binding sites with $0.5 \%$ Tween 20 (Sigma) for 2 hours, the filter was

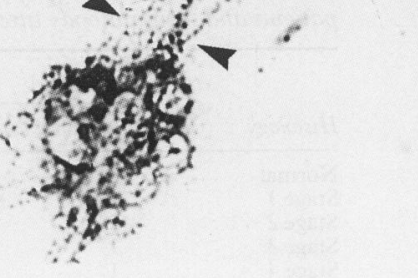




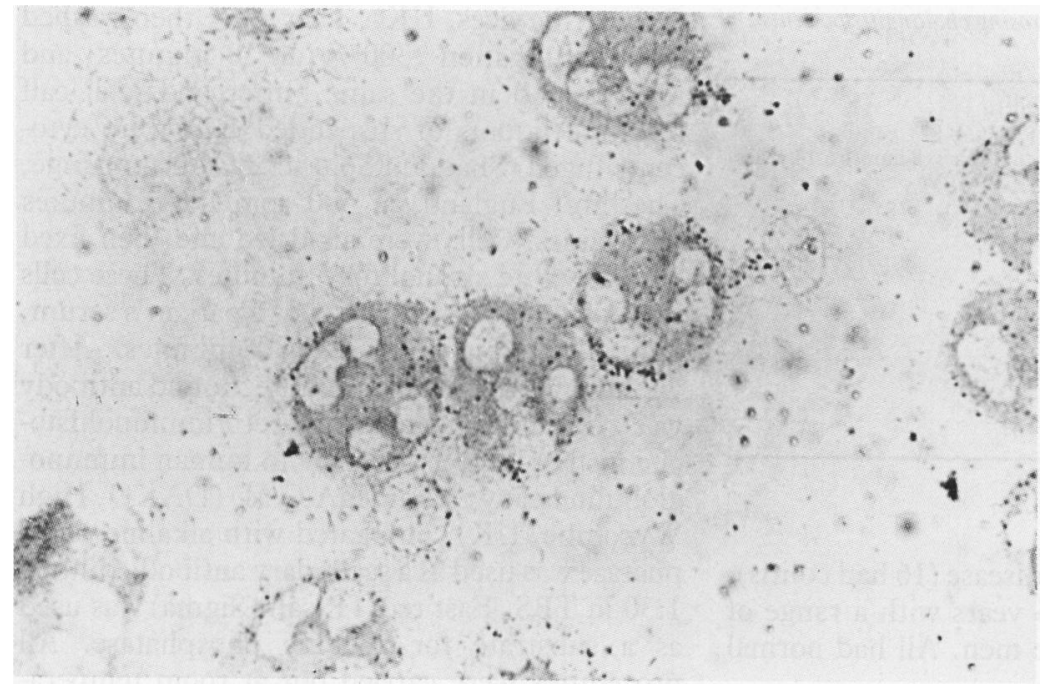

Figure 2: Type 2 pattern of staining - diffuse cytoplasmic staining with no filaments.

Figure 3: Negative staining with serum from a normal subject. Small arrows show outline of the cells. The big arrow indicates the nucleus. incubated with patient's sera (diluted 1:10 in TBS with $0.5 \%$ Tween 20) for 30 minutes, washed in $0.5 \%$ Tween in TBS, and then incubated with the rabbit anti-human immunoglobulin conjugated with alkaline phosphatase for a further 30 minutes. A final wash with $0.5 \%$ Tween 20 for 1 hour was carried out before developing the reaction using fast red. Molecular weight standards (Sigma) transferred on to immobilon were stained with Coomassie blue.

STATISTICAL ANALYSIS

The results were analysed using the $\chi^{2}$ test.

\section{Results}

INDIRECT IMMUNOHISTOLOGICAL STAINING USING ALKALINE PHOSPHATASE METHOD

The following patterns of staining were observed:

(1) Distinct, medium coarse granular cytoplasmic staining concentrated around the nuclear lobes, with filamentous granular staining, forming a network in and around the neutrophil cytoplasm (type 1 pattern) (Fig 1).
The staining frequently obscured the nucleus making assessment of the presence of nuclear staining difficult. However, we interpreted this as being the result of cytoplasmic staining overlying the nucleus, rather than nuclear staining. The cytoplasmic and filamentous granular stainings were always found concomitantly.

(2) Diffuse cytoplasmic staining with no granules or filaments (type 2 pattern) (Fig 2).

(3) A third group of subjects who showed no staining (Fig 3). The results are shown in Table II.

Twenty three $(77 \%)$ of the 30 PSC patients, showed the type 1 pattern of staining. Twenty five of the PSC patients had coexisting ulcerative colitis, of these, 22 showed the type 1 pattern of staining. Ten of the 14 PSC patients who have the HLA B8 DR3 haplotype showed this pattern of staining. One of the three PSC patients who had associated Crohn's disease and hemicolectomy showed this pattern of staining.

The antibody titres within the group of PSC patients were as follows: nine patients had a titre of at least $1: 10$ and 14 patients had a titre of at least $1: 100$. One patient had a positive titre as high as 1:5000. This patient had the highest serum aspartate aminotransferase (940 IU/l) activity of the PSC patients. Liver histology showed expansion of the portal tracts and severe bridging fibrosis (stage 3 ) but the liver was not cirrhotic. He has the HLA B8 DR3 haplotype and an 18 year history of ulcerative colitis which has been quiescent for more than 12 months.

Within the PSC group as a whole, there was no relation between positive staining and the HLA B8 DR3 haplotype $(p>0.05)$. Furthermore, possession of HLA B8 DR3 did not correlate with a higher antibody titre $(p>0.05)$. The antibody titre did not bear any relation to the clinical activity, biochemistry (data not shown), or the liver histology (see Table III) of the patients.

TABLE II Results of the staining patterns observed in disease conditions and in normal subjects

\begin{tabular}{|c|c|c|c|}
\hline \multirow[b]{2}{*}{ Disease state ( $n$ ) } & \multicolumn{2}{|c|}{ Positive } & \multirow[b]{2}{*}{ Negative } \\
\hline & $\begin{array}{l}\text { Typel } \\
\text { pattern }\end{array}$ & $\begin{array}{l}\text { Type } 2 \\
\text { pattern }\end{array}$ & \\
\hline $\begin{array}{l}\text { Primary sclerosing cholangitis } \\
\text { (30) }\end{array}$ & 23 & 0 & 7 \\
\hline Ulcerative colitis (45) & 15 & 14 & 16 \\
\hline $\begin{array}{l}\text { Autoimmune chronic active } \\
\text { hepatitis ( } 3 \text { ) }\end{array}$ & 1 & 2 & 0 \\
\hline Primary biliary cirrhosis (14) & 0 & 5 & 9 \\
\hline Alcoholic liver disease (18) & 0 & 4 & 14 \\
\hline Crohn's disease (32) & 0 & 6 & 26 \\
\hline Coeliac disease (2) & 0 & 1 & 1 \\
\hline $\begin{array}{l}\text { Large duct obstructive jaundice } \\
\text { (6) }\end{array}$ & 0 & 3 & 3 \\
\hline Haemochromatosis (2) & 0 & 0 & 2 \\
\hline Normal (35) & 0 & 2 & $3 \overline{3}$ \\
\hline Total (187) & 39 & 37 & 111 \\
\hline
\end{tabular}

TABLE III Liver histology of primary sclerosing cholangitis patients and their antibody titres

\begin{tabular}{lllll}
\hline & \multicolumn{2}{l}{ Titre } & & \\
\cline { 2 - 5 } Histology & Negative & $1: 10$ & $1: 100$ & $1: 1000$ \\
\hline Normal & 0 & 2 & 2 & 0 \\
Stage 1 & 3 & 1 & 1 & 1 \\
Stage 2 & 2 & 3 & 2 & 0 \\
Stage 3 & 1 & 2 & 2 & 1 \\
Stage 4 & 1 & 0 & 1 & 1 \\
\hline
\end{tabular}




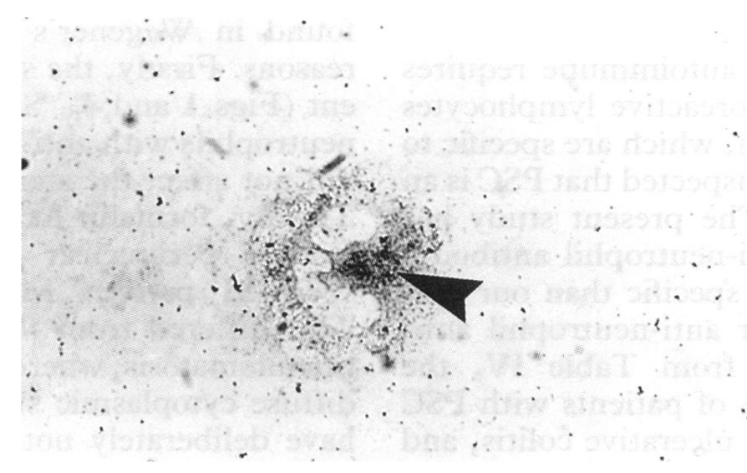

Figure 4: Wegener's granulomatosis pattern of staining with perinuclear accentuation (arrow) and no filaments around the cell.

Fifteen of 45 patients with ulcerative colitis also showed a type 1 pattern of staining. Ten of these 15 patients had active disease but normal serum liver function tests. Among these 15 patients, five had a titre of $1: 10$ and nine a titre of $1: 100$. Only one patient had a titre of $1: 1000$. This particular patient had normal liver function tests and was in remission on sulphasalazine therapy only. She was found to have total colitis on barium enema study.

There was no relation between the disease activity of ulcerative colitis and the presence of anti-neutrophil antibody $(0 \cdot 1>p>0 \cdot 05)$ or the level of anti-neutrophil antibody titre $(p>0.05)$.

The immunoglobulin class of the antineutrophil antibody was almost exclusively IgG in both PSC and ulcerative colitis. No IgM antibody was found. In one case of each condition, IgA only was present. Seven PSC and three ulcerative colitis patients had both IgG and IgA anti-neutrophil antibody.

One patient with chronic active hepatitis was also found to have the type 1 pattern of staining. Otherwise, this type 1 pattern was not observed in any other condition tested.

Sera from eight patients with Wegener's granulomatosis were studied. Of these, two were negative and the other six patients showed granular cytoplasmic staining with perinuclear accentuation, but not nuclear staining and no filamentous staining (Fig 4). It is important to note that this differs from both type 1 (PSC) and type 2 patterns.

COMPARISON BETWEEN ALKALINE PHOSPHATASE AND PEROXIDASE METHODS ON PSC, ULCERATIVE COLITIS, AND CROHN'S DISEASE SERA (TABLE IV) As can be seen from Table IV, the peroxidase method is sensitive in detecting the antineutrophil antibody but it is not specific. Furthermore, we found that the results of the assay seem to depend on the volume of hydrogen

TABLE IV A comparison between the alkaline phosphatase and peroxidase methods in detecting the anti-neutrophil antibody

\begin{tabular}{lll}
\hline & $\begin{array}{l}\text { Alkaline } \\
\text { phosphatase } \\
(\% \text { type 1 pattern })\end{array}$ & $\begin{array}{l}\text { Peroxidase } \\
(\% \text { positive })\end{array}$ \\
\hline Primary sclerosing cholangitis & 77 & 90 \\
Ulcerative colitis & 33 & 67 \\
Crohn's disease & 0 & 61 \\
\hline
\end{tabular}

TABLE V Anti-neutrophil antibody detection using alkaline phosphatase and immunofuorescent (IF) methods

\begin{tabular}{lll}
\hline & $\begin{array}{l}\text { Alkaline } \\
\text { phosphatase } \\
\text { (\% type I pattern) }\end{array}$ & $\begin{array}{l}\text { IF method } \\
\text { (\% positive) }\end{array}$ \\
Disease state & 77 & 86 \\
\hline Primary sclerosing cholangitis (30) & 33 & 83 \\
Ulcerative colitis (45) & 0 & 67 \\
Crohn's disease (32) & 0 & 57 \\
Primary biliary cirrhosis (14) & 0 & 64 \\
Alcoholic liver disease (18) & 33 & 67 \\
Chronic active hepatitis (3) & 0 & 50 \\
Normal (35) & 0 & \\
\hline
\end{tabular}

peroxide used. When $30 \mu \mathrm{l}$ of $3 \%$ hydrogen peroxide were used, most sera were positive and the staining was nuclear. If $100 \mu \mathrm{l}$ were used instead, the staining became more cytoplasmic. When the hydrogen peroxide volume reached $300 \mu \mathrm{l}$, both cytoplasmic and nuclear staining were virtually abolished. For comparison with the alkaline phosphatase technique, we used 100 $\mu l$ of hydrogen peroxide.

\section{ANTI-NEUTROPHIL ANTIBODY DETECTION USING} IMMUNOFLUORESCENT METHOD AND COMPARISON WITH ALKALINE PHOSPHATASE METHOD

Table $\mathrm{V}$ illustrates the results. The immunofluorescent method is again sensitive in detecting the anti-neutrophil antibody but is not specific. The results are comparable with those obtained using peroxidase technique. Eight patients with Wegener's granulomatosis were all positive, showing the characteristic granular, diffuse cytoplasmic immunofluorescence pattern.

\section{NATURE OF THE PSC ANTIGEN}

Preliminary analysis of the antigen has been performed. This has shown that it is heat stable to at least $37^{\circ} \mathrm{C}$. Furthermore, pretreatment of neutrophils with pepsin before performing the immunohistological labelling removes the staining. This suggests that the antigen is proteinaceous in nature.

Preincubation of neutrophils with a mouse monoclonal $\left(\mathrm{IgGl}_{\mathbf{k}}\right.$ ) anti-myeloperoxidase (Dr D Y Mason, University of Oxford) did not affect the staining of PSC sera. Similar treatment abolished positive staining using sera from patients with Wegener's granulomatosis. Formalin fixation of neutrophils changed the pattern of PSC staining to a diffuse cytoplasmic speckled pattern with no nuclear staining. The filamentous staining is not affected.

Colchicine is known to interfere with the microtubules of the neutrophils. ${ }^{20}$ Preincubation of neutrophils for $30-60$ minutes at $37^{\circ} \mathrm{C}$ with colchicine $\left(2.5 \times 10^{-5} M\right.$ to $\left.2.5 \times 10^{-4} \mathrm{M}\right)$ (Sigma) before carrying out the indirect immunohistological labelling did not affect the staining pattern.

RESULTS OF WESTERN BLOTTING

In contrast to the immunohistological staining technique, we could not obtain consistent results despite numerous attempts. Positive bands were generated using PSC sera, but they were not significantly different from those found with non-PSC sera. 


\section{Discussion}

To describe a disease as autoimmune requires the demonstration of autoreactive lymphocytes or autoantibodies, or both, which are specific to that disease. It has been suspected that PSC is an autoimmune disease. ${ }^{1-5}$ The present study has shown a cytoplasmic anti-neutrophil antibody, which seems to be more specific than our previously described nuclear anti-neutrophil antibody. As can be seen from Table IV, the antibody is found in $77 \%$ of patients with PSC and $33 \%$ of patients with ulcerative colitis, and not found in Crohn's disease. In contrast, the nuclear anti-neutrophil antibody is found not only in $90 \%$ of PSC patients, but also in more than $60 \%$ of patients with ulcerative colitis or Crohn's disease. Accordingly, the peroxidase technique was stopped. The variability of patterns resulting from the use of different volumes of hydrogen peroxide also argued against further use of this technique.

It is interesting to note that Duerr et al have also reported a cytoplasmic anti-neutrophil antibody in PSC and ulcerative colitis patients using a different method, namely a fixed cell ELISA screening test followed by indirect immunofluorescent staining. ${ }^{69}$ This showed perinuclear immunofluorescent staining in $69 \%$ of the ulcerative colitis sera, $67 \%$ of PSC sera, and 55\% of PSC without ulcerative colitis sera. They also found a significant percentage (12\%) of patients with Crohn's disease positive by their method. In contrast, none of our patients with Crohn's disease showed a type 1 pattern of staining and only $33 \%$ of ulcerative colitis patients were positive. This suggests that the alkaline phosphatase technique is more specific for PSC than their indirect immunofluorescent method. Indeed, the results obtained with the immunofluorescent method (without previous ELISA screening) showed that it was similar to the peroxidase method. Accordingly, the type 1 pattern obtained with the alkaline phosphatase method seems to be more sensitive and specific for PSC than other available methods. Furthermore, our method has the following advantages. The technique requires only one step, a special (fluorescence) microscope is not required, the result is easy to interpret, and slides can be kept permanently for future reference. We intend, therefore, to use this method for further investigation into its utility in diagnosis of PSC.

While the antigen(s) seems to be proteinaceous, the failure to identify a consistent band on western blotting suggests the antigen(s) may be very labile and therefore destroyed or denatured by the lysis or the western blot techniques. Alternatively, it may be particularly insoluble and not effectively extracted from the cells. Further work is in progress to clarify this problem. Our results with western blotting contrast with those of Klein $e t a l^{5}$ who identified a 65 $\mathrm{kDa}$ band which seemed to be specific for PSC.

The antigen associated with the perinuclear staining pattern (p-ANCA) in Wegener's granulomatosis has been shown to be myeloperoxidase,${ }^{21}$ while the antigen associated with the cytoplasmic staining pattern ( $p$-ANCA) is a serine proteinase. ${ }^{22}$ We do not believe that the anti-neutrophil antibody in PSC is similar to that found in Wegener's granulomatosis for three reasons. Firstly, the staining pattern was different (Figs 1 and 4). Secondly, preincubation of neutrophils with anti-myeloperoxidase antibody did not affect the staining pattern of PSC sera. Thirdly, formalin fixation changed the pattern from a perinuclear to a diffuse cytoplasmic speckled pattern with no nuclear staining. This differed from the situation in Wegener's granulomatosis, where formalin fixation induced diffuse cytoplasmic staining. ${ }^{21}$ Accordingly, we have deliberately not used the terms c-ANCA (anti-neutrophil cytoplasmic antibody exhibiting cytoplasmic pattern) or p-ANCA (antineutrophil cytoplasmic antibody exhibiting perinuclear pattern) in describing the antibody.

It is known that $70 \%$ of PSC patients have coexisting ulcerative colitis, ${ }^{23}$ and about $5 \%$ of ulcerative colitis patients develop PSC. It is of interest, therefore, that ulcerative colitis is the only disease, other than PSC, that also has a significant proportion of patients with a type 1 anti-neutrophil antibody pattern. Long term follow up of these patients is underway to see if any of them develop PSC. If so, detection of antineutrophil antibody may provide a prognostic marker for PSC in ulcerative colitis. Furthermore, this may suggest that these diseases share a pathogenic mechanism. It seems likely that the anti-neutrophil antibody found in PSC is an epiphenomenon. Whether this autoantibody has direct pathogenic importance, as in Wegener's granulomatosis, ${ }^{24} 25$ remains to be determined.

In summary, we have demonstrated the diagnostic importance of an anti-neutrophil antibody in PSC compared with other chronic liver diseases. This may be of particular benefit in paediatric liver diseases where the diagnosis of PSC may be made without resorting to invasive techniques. The prognostic importance of the anti-neutrophil antibody in both PSC and ulcerative colitis remains to be determined, as does the nature of the proteinaceous antigen(s).

We thank Mr J Britton, Dr H Chapel, Dr D Jewell, and Dr J Trowell for supplying some of the sera used in this study and Dr D Mason and Dr K Micklem for technical advice.

The paper was partly presented as an abstract at the British Society of Gastroenterology Autumn Meeting 1991, London.

1 Chapman RW, Varghese Z, Gaul R, Patel G, Kokinon N, Sherlock S. Association of primary sclerosing cholangitis with HLA-B8. Gut 1983; 24: 38-41.

2 Chapman RW, Kelly P, Heryet A, Jewell DP, Fleming KA Expression of HLA-DR antigens on bile duct epithelium in Expression of HLA-DR antigens on bile duct epitheli
primary sclerosing cholangitis. Gut $1988 ; 29: 422-27$.

3 Snook JA, Chapman RW, Fleming KA, Jewell DP. Peripheral blood and portal tract lymphocytes populations in primary sclerosing cholangitis. F Hepatol 1989; 9: 36-41.

4 Snook JA, Chapman RW, Fleming KA, Jewell DP. Antineutrophil nuclear antibody in ulcerative colitis, Crohn's disease and primary sclerosing cholangitis. Clin Exp Immunol 1989; 76: 30-3.

5 Klein R, Eisenburg J, Weber P, Seibold F, Berg PA. Specificity of antibodies against granulocytes detected by western blotting (WB) for the diagnosis of primary sclerosing cholangitis. F Hepatol 1990; 11 (suppl 2): S34.

6 Duerr RH, Targan RS, Landers CJ, et al. Neutrophil cytoplasmic antibodies: A link between primary sclerosing plasmic antibodies: A link between primary sclerosing
cholangitis and ulcerative colitis. Gastroenterology 1991; 100: 1385-91.

7 Hardarson S, LaBrecque DR, Mitros FA, Neil G, Goeken JA. Anti-neutrophil cytoplasmic antibody (ANCA) associated with ulcerative colitis and sclerosing cholangitis. Gastroenterology 1991; 100 (suppl): A585.

8 Saxon A, Shanahan F, Landers C, Ganz T, Targan S. A distinct subset of anti-neutrophil cytoplasmic antibodies is associated with inflammatory bowel disease. $\mathcal{F}$ Allergy Clin Immunol 1990; 86: 202-10.

9 Duerr RH, Targan SR, Landers CJ, Sutherland LR Shanahan F. Anti-neutrophil cytoplasmic antibodies in ulcerative colitis. Comparison with other colitides/diarrhea illness. Gastroenterology 1991; 100: 1590-6. 
10 Pool MO, Ellerbroek PM, Meuwissen SGM, ei al. Serum antineutrophil autoantibodies in patients with inflammatory bowel disease: localization studies of the related antigen(s). Gastroenterology 1991; 100 (suppl): A326.

11 Reumaux D, Colombel JF, Cortot A, Noel LH, Paris JC, Duthilleul $P$. Anti-neutrophil antibodies in sera from patients with inflammatory bowel disease. Gastroenterology 1991; 100 (suppl): A609.

12 Rustgi A, Podolsky D, McCluskey R, Niles J. Antineutrophilic antibodies in sera from patients with inflammaneutrophilic antibodies in sera from patients with inflamma-
tory bowel disease. Gastroenterology $1991 ; 100$ (suppl): A612.

13 Sharma AN, Rabson AR, Kaplan MM. Perinuclear antineutrophil cytoplasmic antibodies (ANCA) in primary sclerosing cholangitis. Hepatology 1991; 14: 64A.

14 Chapman RW, Arborgh BA, Rhodes JM, et al. Primary sclerosing cholangitis: a review of its clinical features, cholangiography, and hepatic histology. Gut 1980; 21: $870-7$.

15 Ludwig J, Barham SS, LaRusso NF, Elveback LR, Wiesner RH, McCall JT. Morphologic features of chronic hepatitis associated with primary sclerosing cholangitis or chronic associated with primary sclerosing cholang
ulcerative colitis. Hepatology $1981 ; 1: 632-40$

16 Wiik A. Delineation of a standard procedure for indirect immunofluorescence detection of anti-neutrophil cytoimmunofluorescence detection of anti-neutrophil
plasmic antibody. APMIS 1989; 97 (suppl 6): 12-3.

17 Ferrante A, Thong YH. A rapid one-step procedure for purification of mononuclear and polymorphonuclear leucocytes from human blood using a modification of the
Hypaque-Ficoll technique. $\mathcal{F}$ Immunol Methods 1978; 24: 389-93.

18 Laemmli U. Cleavage of structural proteins during assembly of the head of bacteriophage T4. Nature 1970; 227: 680-85.

19 Sambrook J, Fritsch EF, Maniatis T. Detection and analysis of proteins expressed from cloned genes. In: Molecular cloning. A laboratory manual. 2nd ed. Cold Spring Harbor, New York: Cold Spring Harbor Laboratory Press, 1989; 18: 64-6.

20 Malawista SE, Bensch KG. Human polvmorphonuclear leukocytes: Demonstration of microtubules and effect of eukocytes: Demonstration of microt

21 Falk R, Jennette JC. Anti-neutrophil cytoplasmic autoantibodies with specificity for myeloperoxidase in patients with systemic vasculitis and idiopathic necrotising and crescentic glomerulonephritis. NEFM 1988; 25: 1651-7.

22 Niles JL, McCluskey RT, Ahmed MF, Arnaout MA Wegener's granulomatosis autoantigen is a novel neutrophil serine proteinase. Blood 1989; 74: 1888-93.

23 Chapman RW. The immunology of primary sclerosing cholangitis. Springer Semin Immunopathol 1990;12: 121-8.

24 Falk RJ, Terell RS, Charles IA, Jennett JC. Anti-neutrophi! cytoplasmic autoantibodies induce neutrophils to degranucytoplasmic autoantibodies induce neutrophils to degranu1990;87: 4115-9.

25 Haydek J, Parveen S, List T, Doria M, Keshavarzian A Reactive oxygen metabolites in experimental colitis: the effect of catalase. Gastroenterologv 1991; 100 (suppl): A585. 